EDITORIAL

\title{
Role of Anti-Aging QOL Common Questionnaire in Clinical Medicine
}

\author{
Hiroshi Bando* \\ Medical Research, Tokushima University, Japan
}

\section{Keywords}

Anti-aging medicine, anti-aging QOL (AAQOL), common questionnaire, activities of daily living (ADL), quality of life (QOL), Japanese Society of Anti-Aging Medicine (JSAAM)

\section{Editorial}

Anti-aging medicine has been a popular treatment strategy worldwide since its inception. ${ }^{1}$ Primarily, anti-aging medicine is used to improve the patients' quality of life (QOL), promote healthy activities of daily living (ADL), and to protect from and treat various diseases such as various metabolic syndromes. ${ }^{2}$

Factors, such as nutrition and dietary habits, physical activity and exercise, rest, alcohol drinking, tobacco smoking, and oral health influencing health and longevity are considered before diagnosis. These factors are fundamental for improving the patient's quality of life and life-style. This concept was announced as a fundamental direction of "Health Japan 21" for comprehensive implementation of national health promotion by the Ministry of Heath, Labour and Welfare, Japan. ${ }^{3}$ However, the recent slogan is to promote and extend healthy life expectancy rather than increase of life expectancy until 2022.

From the perspective of anti-aging, the degree of aging process is evaluated through five fundamental elements/axes:axes of muscle, vascular, neural, hormone, and bone ages. ${ }^{4}$ The ideal path to healthy and long life would be the tendency of a balanced aging for all the axes. Any dysfunction in any organ or axis of the body requires treatment in light of the anti-aging medicine concept.

Open Access

Further, there are various factors that are central for the evaluation. They include immunological stress, oxidative stress, ${ }^{5}$ glycative stress, ${ }^{6}$ psychosomatic stress, and sedentary/unhealthy lifestyle. By judging the degree of each risk factor, the priority of the treatments could be determined.

The QOL questionnaire has been used for various purposes. The Japanese Society of AntiAging Medicine (JSAAM) has developed the "Anti-Aging QOL Common Questionnaire", which has now called as AAQOL questionnaire. The aim of AAQOL questionnaire is to improve the quality of anti-aging medical checkups.

To validate its efficacy and relevancy, it is imperative that of AAQOL questionnaire be tested Accepted: August 18, 2018 Published: August 29, 2018

Copyright: $\odot 2018$ Bando H. This is an open access article distributed under the terms of the Creative Commons Attribution License, which permits unrestricted use, distribution, and reproduction in any medium, provided the original author and source are credited.

Corresponding author:

Hiroshi Bando, Medical Research, Tokushima University, Tokushima, Japan Email: pianomed@bronze.ocn.ne.jp using the functional age data calculated through various tests. ${ }^{7}$ Functional age data include several axes, such as muscle, vascular, neural, hormone, and bone.

A study was conducted ( $N=5827$ ) to test its validity. ${ }^{7}$ From the results, it was observed that the following items tend to increase with aging: palpitation, shortness of breath, gray hair, hair loss, tinnitus, arthralgia, frequent urination in $7 / 33$ physical symptoms, shallow sleep, difficulty in falling asleep, and lapse of memory in $3 / 21$ mental symptoms. Results of the structural equation modeling (SEM) analysis suggested that lifestyle-related items influence the body and the mentality. Exercise was identified as the most influential lifestyle factor.

The current version of the AAQOL questionnaire includes 33 physical and 21 mental symptoms, with each evaluation based on a 5-point scale. Further, the questionnaire also included sleep/exercise habits, smoking, and drinking as lifestyle-related questions. The questionnaire was arranged taking into account the Theory of Yin-Yang and the Five Elements. 
We have investigated several analyses methods for AAQOLquestionnaire, ${ }^{8}$ which included categorization of similar symptoms in over 50 items. Initially, we used four items for the first group and conducted analytical assessment using eight categories, with seven items each. The categories are as follows ${ }^{8}$ :

1. VDT-related symptoms: tired eyes, blurry eyes, eye pain, stiff shoulders, muscle pain/strain, lethargy, headache

2. Fatigue-related symptoms: overweight, lethargy, no feeing of good health, appetite loss, early satiety, epigastralgia

3. Persistent neurological symptoms: palpitation, thirst, headache, dizziness, tinnitus, lumbago, arthralgia

4. Depression-related symptoms: irritability, short temper, reluctance to talk, depression, feeling of uselessness, shallow sleep, difficulty falling asleep

5. Loss of self-confidence: loss of motivation, no feeling of happiness, nothing to look forward to, daily life is not enjoyable, loss of confidence, pessimism, anxious before sleeping

6. Anxiety-related symptoms: lapse of memory, inability to concentrate, inability to solve problems, inability to decide, a sense of tension, anxiety without reasons, vague feeling of fear

7. Autonomic nerve-related symptoms: dizziness, tinnitus, edema, sweating, frequent urination, hot flush, cold sensation

8. Fragile constitution: weight loss, skin problems, weak chest, coughing and sputum, diarrhea, constipation, cold sensation

For decades, numerous questionnaires have been developed and used. ${ }^{9,10}$ Among them, short form-36 (SF-36) has been widely used for incidating a rating scale for health-related QOL. ${ }^{9}$ Besides, there have been several other questionnaires, such as the WHO/QOL-26 developed by the World Health Organization (WHO) and EuroQol (EQ-5D) Health Scores. ${ }^{11}$ Some of the questionnaires were developed a long time ago, and thus are in use for clinical studies. This could be attributed to the rather difficult procedure and analysis method associated with these questionnaires.

AAQOL questionnaire is clinically simple and easy to evaluate, and thus it has been used for clinical research, for example Masters Athletes for physical, psychological, and lifestyle habits. ${ }^{12}$ AAQOL questionnaire is primarily useful for medical check-up, especially for identifying dysfunctional organ system(s). In Japan, the anti-aging medical checkup is called as "Human Dock", which is focused on assessing the degree of aging utilizing AAQOL questionnaire. ${ }^{13}$ The usefulness and the effectiveness of AAQOL questionnaire were validated following the analysis of the data obtained as a result of numerous researches. ${ }^{7,13 .}$

In summary, the management for anti-aging medicine is necessary in a clinical setting. ${ }^{14,15}$ From such standpoint, it is imperative that a convenient and useful questionnaire is developed and used. AAQOL questionnaire is not only useful in medicine, but also finds application in food, cosmetic, and health industries, exercise equipment, sports, education, and occupational hygiene. It is expected that $A A Q O L$ questionnaire would find application in different clinical studies and help contribute to the development of clinical research in future.

\section{References}

1. Rowe JW, Kahn RL. Human aging: usual and successful. Science.1987; 237(4811): 143-149

2. Vaiserman A, Lushchak O. Implementation of longevity-promoting supplements and medications in public health practice: achievements, challenges and future perspectives. J Trans/ Med. 2017; 15(1):160. doi: 10.1186/s12967-017-1259-8.

3. https://www.mhlw.go.jp/file/06-Seisakujouhou-10900000-Kenkoukyoku/0000047330.pdf

4. Yonei $Y$, Takahashi $Y$, Watanabe $M$, et al. Effects on the human body of a dietary supplement containing I-carnitine and Garcinia cambogia extract: A study using double-blind tests. J Clin Biochem Nutr. 2008; 42: 89-103. 
5. Naito Y, Lee CM, Kato Y, Nagai R, Yonei Y. Oxidative stress markers. Anti-Aging Medicine. 2010; 7: $36-44$.

6. Barić N. Role of advanced glycation end products in Alzheimer's disease. Glycative Stress Res. 2014; 1:68-83.

7. Oguma Y, lida K, Yonei Y, Satoh T. Significance evaluation of Anti-Aging QOL Common Questionnaire. Glycative Stress Res.2016; 3(3): 177-185. doi:10.24659/gsr.3.3_177.

8. Bando H, Yoshioka T, Yonei Y, Nakamura T. Investigation of quality life in athletes from an anti-aging perspective. Primary Care Japan. 2006; 4(1):47-51.

9. Ware JE.SF-36 Health Survey manual and interpretation guide. Boston, MA: New England Medical Centre, 1993.

10. http://www.mhlw.go.jp/toukei/list/dl/20-21-h25.pdf.

11. EuroQoL Group. EuroQoL: A new facility for the measurement of health-related quality of life. Health Policy. 1990; 16: 199-208.

12. Bando H, Takenaka Y, Nakamura T, Kounoike K, Yonei Y. Investigation for Quality of Life (QOL) and self-esteem for health in masters' athletes. Glycative Stress Res. 2015; 2(4): 174-181. doi: 10.24659/gsr.2.4_174.

13. Yonei Y, Takabe W. Aging assessment by anti-aging medical checkup. Health Evaluat Prom. 2015; 42 : 459-464.

14. Longo VD, Antebi A, Bartke A, et al. Interventions to Slow Aging in Humans: Are We Ready? Aging Cell. 2015; 14(4):497-510. doi: 10.1111/acel.12338.

15. Pan H, Finkel T. Key proteins and pathways that regulate lifespan. J Biol Chem. 2017; 292(16): 6452-6460. doi: 10.1074/jbc.R116.771915. 